\title{
Radiative Heat Transfer in Participating Medium and Dynamic Region Monte Carlo Method by Region Adaption
}

\author{
Qing Ai, Hua Liu, Xinlin Xia, Chuang Sun, and Ming Xie \\ School of Energy Science and Engineering, Harbin Institute of Technology, 92 West Dazhi Street, Harbin 150001, China \\ Correspondence should be addressed to Qing Ai; hitaiqing@hit.edu.cn and Xinlin Xia; xiaxl@hit.edu.cn
}

Received 21 August 2014; Accepted 21 October 2014

Academic Editor: Yong Huang

Copyright ( 2015 Qing Ai et al. This is an open access article distributed under the Creative Commons Attribution License, which permits unrestricted use, distribution, and reproduction in any medium, provided the original work is properly cited.

\begin{abstract}
A dynamic region Monte Carlo method (DRMC) is proposed to simulate radiative heat transfer in participating medium. The basic principle and solution procedure of this method is described; radiative heat transfer in a two-dimensional rectangular region of absorbing, emitting, and/or scattering gray medium is analyzed. A comparison between DRMC and the traditional Monte Carlo method (TMC) is investigated by analyzing the simulated temperature distribution, the computing time, and the number of the sampling bundles. The investigation results show that, to compare with TMC, the DRMC can obviously reduce the computing time and storage capacity under the same solution precision for radiative transfer in optically thick medium; the DRMC allows bypassing the difficulties encountered by TMC in the limit of optically thick extinction.
\end{abstract}

\section{Introduction}

Radiative heat transfer plays an important role in heat transfer process, especially in some high temperature applications. Compared with other heat transfer modes, there are a lot of fundamental difficulties of radiative heat transfer due to its different characteristics [1]. Radiative heat transfer in participating medium is described by radiative transfer equation (RTE), which is an integrodifferential equation, the common numerical method for solving this equation, such as finite volume method (FVM) and finite element method (FEM), relies on various degrees of approximation, and this makes it complex and difficult to solve with accuracy.

The Monte Carlo (MC) method has been widely used to numerically simulate radiative heat transfer in participating medium [2]; because of the flexibility of this method, it is known to be very good in dealing with radiative heat transfer with complex geometries and/or complex spectral properties; in addition, due to the high solution precision of this method, the MC predicted results are usually regarded as benchmark solutions $[3,4]$. As a statistical method, a standard deviation of the MC simulated results can be computed, and that may be interpreted as a numerical uncertainty [5].
However, if the medium is optically thick, a large proportion of the emitted bundles will be quickly absorbed by the medium in the vicinity of their emission positions; it indicates that only a few bundles participate in distant radiative transfer; therefore, a large number of bundles are required in order to get a believable statistical solution. In addition, more grid numbers are needed in optically thick medium in order to get high precision solutions. Therefore, the TMC method would face the difficulties of long computing time, large storage capacity, and the handicap of optically thick limit when simulating radiative transfer in participating medium $[6,7]$.

Some researchers give up high precision prediction; they use some approximate methods to solve radiative transfer in participating medium. Diffusion approximation formulation is one of the most widely used method for solving radiative transfer in optically thick medium [8-10]; the calculated results are acceptable in some practical applications; however, this formulation is just suitable for isotropic scattering medium. Enguehard [11] developed a modified diffusion approximation formulation called "non-gray anisotropically scattering Rosseland approximation," which expands it in anisotropically scattering medium, but the modified diffusion approximation formulation still encounters the problem of 
low precision calculation; it may not be used in some special applications. Duan and Min [12] proposed a semianalytic technique to speed up successive order of scattering model for optically thick media. Amosov et al. [13] solved radiative transfer in an optically thick slab from integral radiative transfer equation by using an approximate solution method. These approximation methods are efficient but cannot solve with high precision.

Based on TMC, some modified Monte Carlo methods were proposed to solve radiative transfer in optically thick medium. An early hybrid transport-diffusion method called random walk (RW) [14, 15] was proposed for improving the efficiency of MC simulation, which can be effective in radiative transfer simulation in optically thick medium. In recent years, another hybrid method called discrete diffusion Monte Carlo (DDMC) [16-18] was developed for increasing the efficiency of MC simulations in optically thick medium; this method can be more efficient than RW. Some other researchers proposed a net-exchange Monte Carlo approach $[5,7,19]$ which is based on a net-exchange formulation and on adapted optical path sampling procedures carefully designed, and it ensures satisfactory convergence over a wide range of absorption and scattering optical thicknesses; however, the proposed algorithm still encounters a convergence difficulty in the case of optically thick and highly scattering media.

In this paper, a new method called dynamic region Monte Carlo method (DRMC) is proposed to analyze radiative heat transfer in a two-dimensional rectangular region of participating medium with a large optical thickness; the medium can be absorbing only or absorbing-scattering. The computing precision and computing time are analyzed by comparing with the results predicted by TMC method.

The rest of this paper is organized as follows: in Section 2, the basic principle and numerical model of DRMC are presented; some related mathematical formulations are also given in this section. Section 3 discusses our results predicted by DRMC and/or TMC; the medium can be absorbing only or absorbing-scattering, and in this section, a detailed comparison between DRMC and TMC is investigated by analyzing the numerical predicted temperature field and computing time in a two-dimensional rectangular medium. At last, we end with a conclusion in Section 4.

\section{Basic Principle and Numerical Model}

To solve radiative heat transfer in participating medium, it is usually required to integrate incident radiation from all the discrete surface and volume elements. Compared with other numerical methods, MC method can easily deal with scattering term of the radiative transfer equation; the mathematical model is simple. However, if the medium is optically thick, a lot of computing nodes are needed to get a high precision calculation result, and this leads to a large amount of computing time and data capacity.

The basic principle of DRMC is to combine advantages of partition allocation method $[20,21]$ and the TMC method. In DRMC, the sample tracking domain is limited to a rectangular subdomain within a certain optical thickness

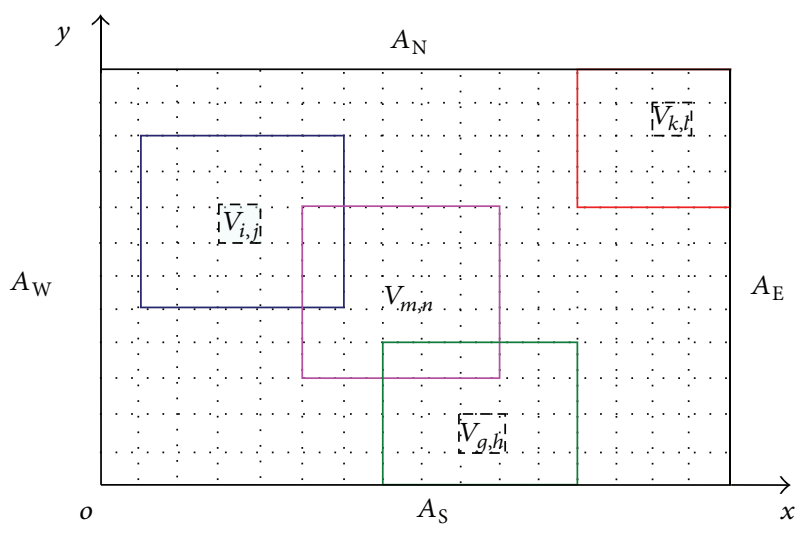

FIgURE 1: Partition schematic diagram of DRMC.

centered about the particle's current position, instead of the entire computing space (computational domain). Radiative transfer in the subdomain is directly simulated by $\mathrm{MC}$ method, while the thermal radiation energy transmit to the discrete elements outside of the subdomain can be equivalently calculated by setting an equivalent temperature region reasonably. In the DRMC, for each sample unit, there is a corresponding tracking domain, and the tracking domain moves with the position of sampling unit, so this method is called dynamic region Monte Carlo method.

Figure 1 shows the partition schematic diagram of DRMC; a two-dimensional rectangular medium is taken as example to describe the basic rules of DRMC. Assume that the two-dimensional enclosed medium is divided into $M \times$ $N$ units (volume elements and surface elements), for each volume element $V_{i, j}$ or surface element $A_{i, j}, i \in[1, M], j \in$ $[1, N]$. The subdomain space is attached on the discrete grids of the whole computational domain, for subdomain space $\Omega_{i, j}^{z}$ of element $V_{i, j}$; its coordinate origin is the node coordinates of the element $V_{i, j}$, just as Figure 2(a) has shown; Figure 2(a) also shows the schematic diagram of node distribution for subdomain space $\Omega_{i, j}^{z}$. Subdomain space $\Omega_{i, j}^{z}$ includes $\left(m_{z}^{-}+\right.$ $\left.1+m_{z}^{+}\right) \times\left(n_{z}^{-}+1+n_{z}^{+}\right)$nodes, for each volume element $V_{i, j}^{z}$ or boundary surface element (artificial black body surface element or real surface element) $S_{i, j}^{z}$ in the subdomain, $i \in$ $\left[-m_{z}^{-}, m_{z}^{+}\right], j \in\left[-n_{z}^{-}, n_{z}^{+}\right]$.

Figure 2(b) shows the schematic diagram of the subdomain space $\Omega_{i, j}^{z}$ and its boundary domains; the optical thickness of the subdomain is $2 \tau_{z}$, while the optical thickness of the boundary domain is $\tau_{b}$.

Based on Bouguer's law in participating medium, radiation intensity exponential decay along the route

$$
I_{\lambda}(S)=I_{\lambda}(0) \exp \left[-\int_{0}^{S} \kappa_{e \lambda}\left(S^{*}\right) d S^{*}\right] \text {, }
$$

where $\kappa_{e \lambda}$ is extinction coefficient, $S$ is the propagation distance, and $I_{\lambda}$ is spectral radiation intensity. If $\kappa_{e \lambda}$ is constant

$$
\frac{I_{\lambda}(S)}{I_{\lambda}(0)}=\exp \left[-\kappa_{e \lambda} S\right]=\exp \left[-\tau_{\lambda}\right]
$$




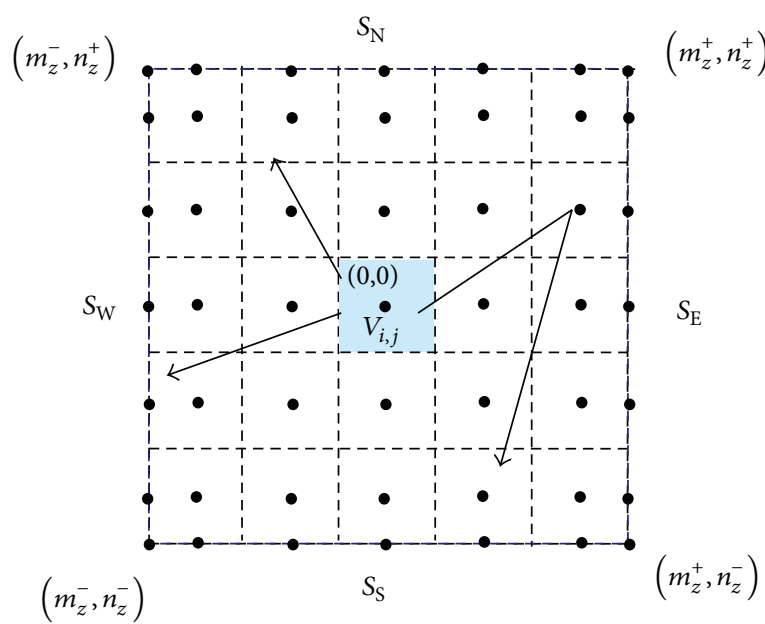

(a)

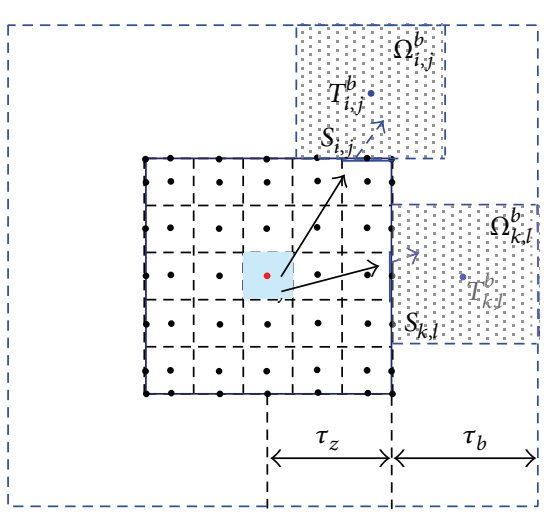

(b)

FIGURE 2: (a) Schematic diagram of node distribution for subdomain. (b) Schematic diagram of computational domain.

The subdomain optical thickness can be determined by the following formulation:

$$
\tau_{\lambda, z}=-\ln \left[\frac{I_{\lambda}^{\prime}(S)}{I_{\lambda}^{\prime}(0)}\right]=-\ln \left[P_{s}\right]
$$

where $P_{s}$ is the proportional coefficient of radiation attenuation, which is used to set the range of the subdomains.

In the coordinate system of the entire spatial domain, the subdomain contains the space of

$$
\Omega_{i, j}=\sum_{\Omega_{i, j}} V_{m, n} \cup \sum_{\Omega_{i, j}}\left(\delta_{m, n} S_{m, n}+\delta_{m, n}^{\prime} A_{m, n}\right),
$$

where $S_{m, n}$ is boundary surface element; $A_{m, n}$ is real boundary surface element, for any volume element $V_{m, n}$ or boundary surface element $S_{m, n}, m \in\left[m+m_{z}^{+}-1, m-m_{z}^{-}+1\right], n \in$ $\left[n+n_{z}^{+}-1, n-n_{z}^{-}+1\right] ; \delta_{m, n}$ and $\delta_{m, n}^{\prime}$ are used to identify artificial boundary surface and real surface; if the boundary surface of the computational subdomain is a real one, $\delta_{m, n}=0$ and $\delta_{m, n}^{\prime}=1$; else if the boundary surface is an artificial blackbody surface, $\delta_{m, n}=1$ and $\delta_{m, n}^{\prime}=0$.

The effective temperature $T^{b}$ of artificial blackbody boundary surface $S_{k, l}$ is the average radiative temperature of the boundary domain nodes. For boundary domain $\Omega_{k, l}^{b}, T_{k, l}^{b}$ can be expressed as

$$
T_{k, l}^{b}=\left[\frac{\sum_{\Omega_{k, l}^{b}} 4 \kappa_{a} V_{i, j} \sigma T_{i, j}^{4}+\delta_{i, j} \sum_{\Omega_{k, l}^{b}} \varepsilon_{i, j} A_{i, j} \sigma T_{i, j}^{4}}{\sum_{\Omega_{k, l}^{b}} 4 \kappa_{a} V_{i, j} \sigma+\delta_{i, j} \sum_{\Omega_{k, l}^{b}} \varepsilon_{i, j} A_{i, j} \sigma}\right]^{1 / 4} .
$$

DRMC just solves radiative heat transfer by MC method in the subdomain; therefore, in order to save storage capacity, the computer just stores the predicted radiation exchange factor of the subdomain:

$$
\begin{aligned}
\operatorname{RD} & {[(M, N),(M, N)] } \\
& \longrightarrow \operatorname{RD}\left[(M, N),\left(-m_{z}^{-}: m_{z}^{+},-n_{z}^{-}: n_{z}^{+}\right)\right] .
\end{aligned}
$$

In $\mathrm{RD}\left[(M, N),\left(-m_{z}^{-}: m_{z}^{+},-n_{z}^{-}: n_{z}^{+}\right)\right],(M, N)$ is system coordinate, while $\left(-m_{z}^{-}: m_{z}^{+},-n_{z}^{-}: n_{z}^{+}\right)$is the local subdomain coordinate. Radiative energy absorbed by any unit $(i, j)$ in the whole computational domain can be expressed as

$$
\begin{aligned}
\phi_{i, j}^{\alpha}= & \sum_{k, l}^{M_{V}} 4 \kappa_{a_{k+i, l+j}} V_{k+i, l+j} \sigma T_{k+i, l+j}^{4} \mathrm{RD}_{k+i, l+j \rightarrow-k,-l} \\
& +\sum_{k, l}^{M_{S}} \delta^{\prime} \varepsilon_{k, l} A_{k, l} \sigma T_{k+i, l+j}^{4} \mathrm{RD}_{k+i, l+j \rightarrow-k,-l} \\
& +\sum_{k, l}^{M_{S}} \delta S_{k, l} \sigma\left(T_{k+i, l+j}^{b}\right)^{4} \mathrm{RD}_{k+i, l+j \rightarrow-k,-l},
\end{aligned}
$$

where $M_{V}$ and $M_{S}$ are the sum of the volume elements and boundary surface elements of the subdomain $\Omega_{i, j}$. The first term on the right is the energy emitted by the volume elements in the computational domain and then absorbed by unit $V_{i, j}$; the second term on the right stands for the energy emitted by the surface elements and then absorbed by unit $V_{i, j}$ if the boundary surfaces are real ones, while the third term is the absorbed energy by unit $V_{i, j}$ which is emitted from the effective imaginary blackbody surfaces, and in fact, it stands for the energy transmit into the subdomain and absorbed by unit $(i, j)$ from other units outside of the subdomain.

Radiative equilibrium implies that anywhere within the media the material absorbs precisely as much radiative energy as it emits; therefore, the radiative equilibrium temperature $T_{i, j}$ inside the media can be determined by keeping track of 


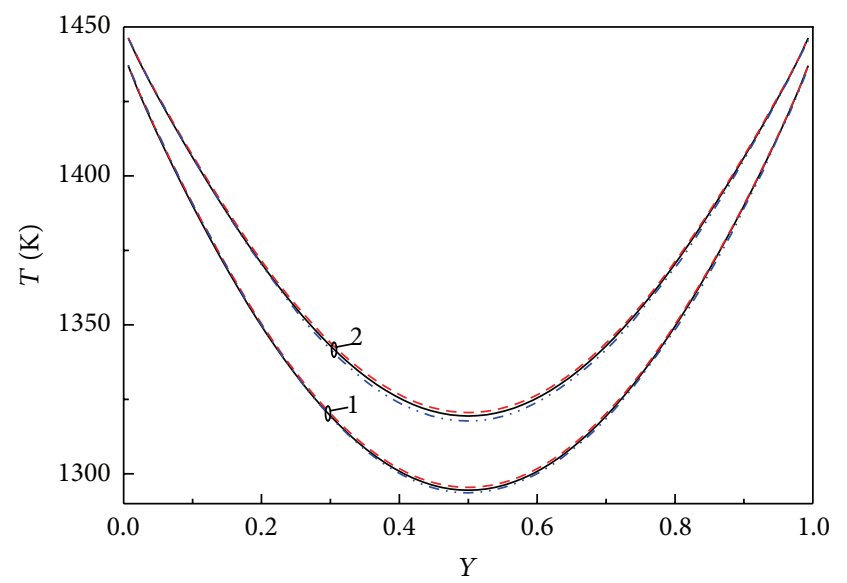

$$
\begin{aligned}
-N_{r} & =10^{6} \\
--- & N_{r}=10^{5} \\
\cdots-N_{r} & =10^{4}
\end{aligned}
$$

(a) Curve $1: X=0.3 ; 2: X=0.5$

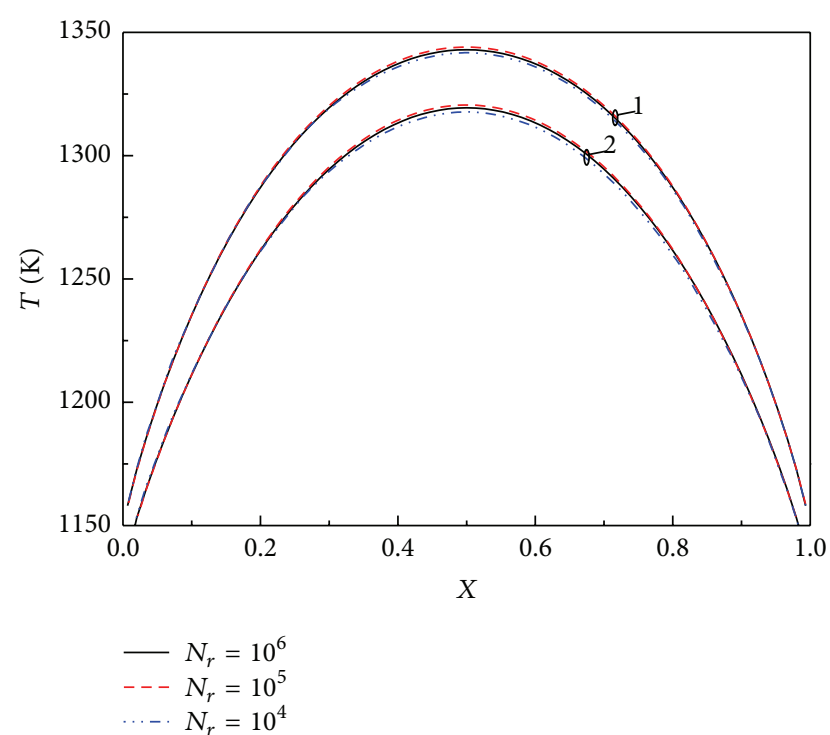

(b) Curve $1: Y=0.3 ; 2: Y=0.5$

FIgURE 3: Temperature field in absorbing medium predicted by TMC method.

the total reemitted energy form unit $(i, j)$. If unit $(i, j)$ is a volume element

$$
\begin{gathered}
\phi_{i, j}^{\alpha}=4 \sigma \kappa_{a_{i, j}} T_{i, j}^{4} V_{i, j}, \\
T_{i, j}=\left(\frac{\phi_{i, j}^{\alpha}}{4 \sigma \kappa_{a_{i, j}} V_{i, j}}\right)^{1 / 4} .
\end{gathered}
$$

Else if unit $(i, j)$. is a surface element, then

$$
\begin{gathered}
\phi_{i, j}^{\alpha}=\varepsilon_{i, j} A_{i, j} \sigma T_{i, j}^{4}, \\
T_{i, j}=\left(\frac{\phi_{i, j}^{\alpha}}{\varepsilon_{i, j} A_{i, j} \sigma}\right)^{1 / 4} .
\end{gathered}
$$

\section{Results and Discussion}

In order to provide a final numerical solution to compare and analyze the results conveniently, the total number of discrete grids of the entire computational domain is in the acceptable range of the TMC method. DRMC and TMC are separately used to solve the radiative equilibrium temperature field of two-dimensional rectangular participating medium; the calculated results and computing time are compared and analyzed by TMC and DRMC in different range of subdomain, boundary domain, and sampling bundles.

The absorption coefficient, scattering albedo, and other radiative properties are assumed constant. The number of discrete grids is $M \times N=77 \times 77$, the length and width of the region are $L_{x}=L_{y}=L$, the optical thickness of the medium is defined as $\tau=\kappa_{e} L_{x}=\kappa_{e} L_{y}=15$, four boundaries are diffuse gray walls with constant emissivity of $\varepsilon=0.5$, the temperature of boundaries is $T_{W E}=T_{W W}=1000 \mathrm{~K}$, $T_{W N}=T_{W S}=1500 \mathrm{~K}$, the entire rectangle medium region is uniformly discrete, and the dimensionless coordinate is defined as $X=x / L_{x}, Y=y / L_{y}$.

3.1. Absorbing-Emitting Medium without Scattering. For different subdomain and boundary domain space, the radiative equilibrium temperature field of absorbing medium with extinction coefficient $\kappa_{e}=15$ and scattering albedo $\omega=0$ are comparatively analyzed by DRMC.

\subsubsection{Analysis of Subdomain and Its Boundary Domain.} Figure 3 shows the temperature distribution of the absorbing medium predicted by TMC method with different sampling bundles; it can be seen that the temperature field has converged when the number of sampling bundles is $N_{r}=10^{5}$, so we consider that the temperature field calculated by TMC is the final numerical solution when the sampling number of the unit is $N_{r}=10^{5}$.

Figure 4 shows the radiative equilibrium temperature field predicted by TMC and DRMC with different subdomain and boundary domain optical thickness.

Figure 4(a) shows the comparison of predicted temperature field by DRMC and the final numerical solution with subdomain optical thickness of $\tau_{z}=5$ and different boundary domain $\tau_{b}$; it can be seen that when $\tau_{b}=0.2$, the temperature field predicted by DRMC agrees very well with the final numerical solution, while when $\tau_{b}$ increase to 0.5 , the predicted temperature field by DRMC deviates from the final solution; this is due to the radiation transmit through the absorbing medium with optical thickness of 5; almost all of the radiative energy have been attenuated, the artificial blackbody boundary surface just influenced by the neighboring units; an increase of boundary domain optical thickness conversely increases calculation error of boundary equivalent blackbody temperature $T^{b}$. 


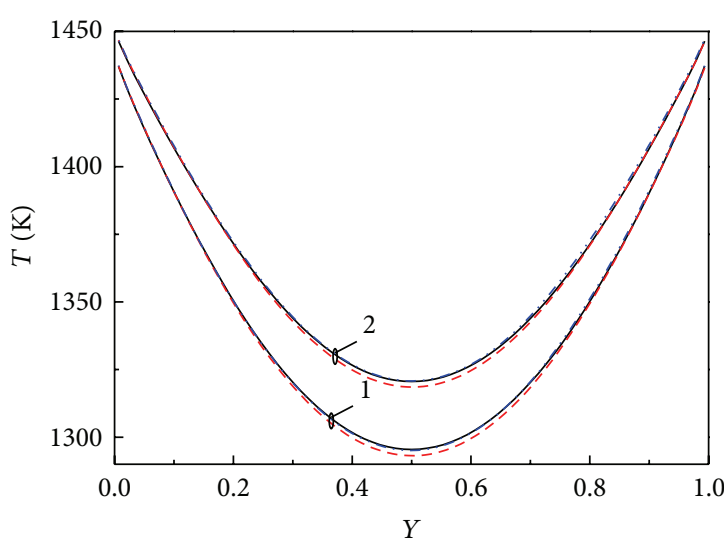

- TMC

… DRMC, $\tau_{b}=0.2$

--- DRMC, $\tau_{b}=0.5$

(a) $\tau_{z}=5.0$

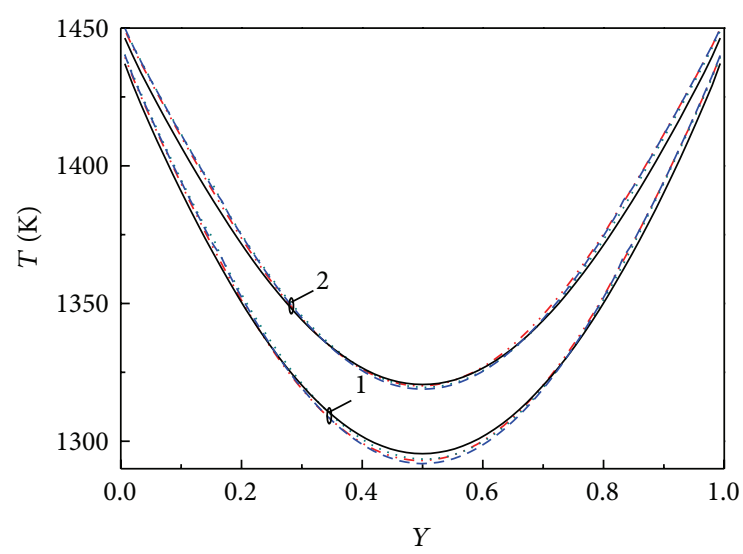

- TMC

--- DRMC, $\tau_{b}=2$

DRMC, $\tau_{b}=0.5$

DRMC, $\tau_{b}=0.2$

(c) $\tau_{z}=2.5$

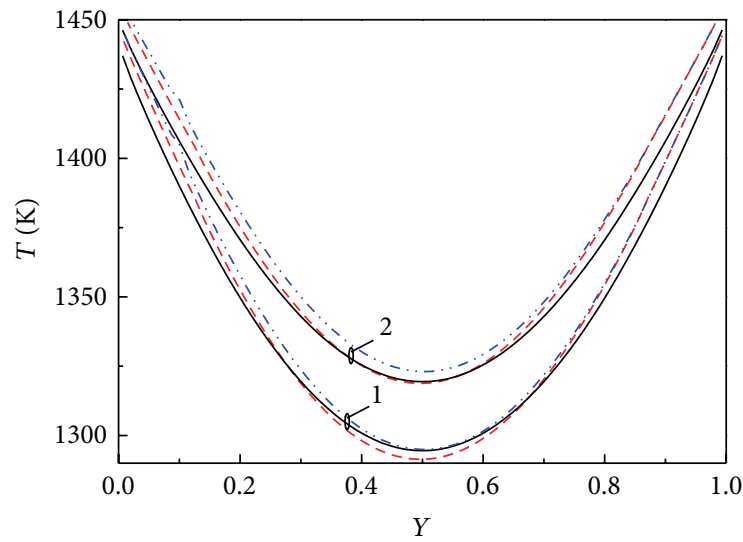

- TMC

- - D DRMC, $\tau_{b}=0.5$

(e) $\tau_{z}=1.5$

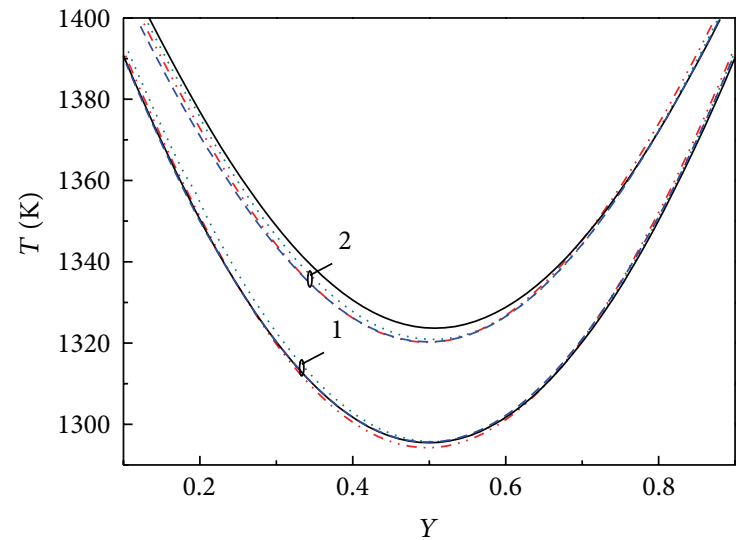

- TMC

-- DRMC, $\tau_{b}=2$

DRMC, $\tau_{b}=0.5$

DRMC, $\tau_{b}=0.2$

(b) $\tau_{z}=3.0$

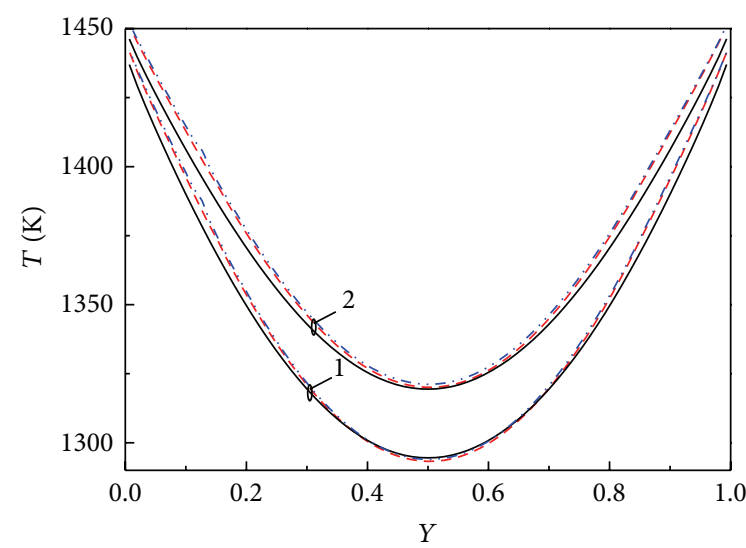

- TMC

-... DRMC, $\tau_{b}=0.2$

-- DRMC, $\tau_{b}=0.5$

(d) $\tau_{z}=2$

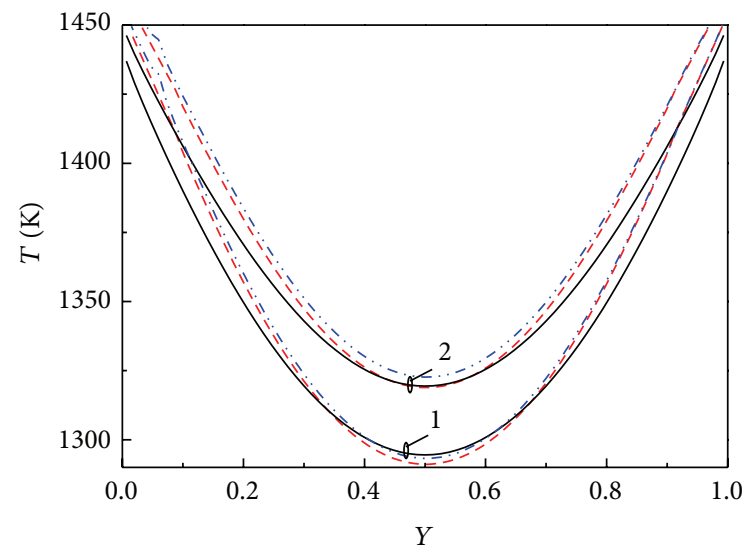

- TMC

- ... DRMC, $\tau_{b}=0.2$

-- DRMC, $\tau_{b}=0.5$

(f) $\tau_{z}=1$

FIgURE 4: Comparison of temperature fields in absorbing medium predicted by TMC and DRMC, Curve $1: X=0.3 ; 2: X=0.5$. 
TABLE 1: Comparison of computing time between TMC and DRMC in absorbing medium.

\begin{tabular}{|c|c|c|c|c|}
\hline Method & Sampling number & $\tau_{z}$ & $\tau_{b}$ & Computing time \\
\hline TMC & $N_{r}=10^{5}$ & & & $86 \mathrm{~min}$ and $36 \mathrm{~s}$ \\
\hline \multirow{6}{*}{ DRMC } & \multirow{6}{*}{$N_{r}=10^{5}$} & 5.0 & 0.2 & $58 \mathrm{~min}$ and $09 \mathrm{~s}$ \\
\hline & & 3.0 & 0.2 & $41 \mathrm{~min}$ and $15 \mathrm{~s}$ \\
\hline & & 2.5 & 0.2 & $37 \mathrm{~min}$ and $12 \mathrm{~s}$ \\
\hline & & 2.0 & 0.2 & $31 \mathrm{~min}$ and $55 \mathrm{~s}$ \\
\hline & & 1.5 & 0.2 & $28 \mathrm{~min}$ and $52 \mathrm{~s}$ \\
\hline & & 1.0 & 0.2 & $25 \mathrm{~min}$ and $36 \mathrm{~s}$ \\
\hline
\end{tabular}

In Figure 4(b), when the subdomain thickness decreased to $\tau_{z}=3$, the temperature field predicted by DRMC of different boundary domain basically agrees with the final numerical solution, the deviation increases with the reduction of $\tau_{b}$, and the maximum deviation does not exceed $0.29 \%$.

Figure 4(c) shows the comparison between the temperature field predicted by TMC and DRMC with different boundary domain and subdomain optical thickness of $\tau_{z}=$ 2.5; the maximum deviation is $0.73 \%$, and at this time, $\tau_{b}$ $=2.0$; it indicates that the scope of neighborhood is not the bigger, the better.

In Figure $4(\mathrm{~d})$, it can be seen that when $\tau_{z}=2$, according to setting $\tau_{b}$ reasonably, the deviation of temperature field predicted by DRMC and TMC does not exceed $0.75 \%$, and the maximum temperature difference is less than $8.6 \mathrm{~K}$.

In Figures 4(e) and 4(f), as the subdomain optical thickness decreases to 1.5 and 1.0, a large deviation exists between the final numerical solution and the temperature field predicted by DRMC, and no matter how $\tau_{b}$ changed, the deviation is still over $1 \%$, the maximum deviation separately reaches $1.35 \%$ and $1.55 \%$, and the maximum temperature differences reach $15.79 \mathrm{~K}$ and $15.69 \mathrm{~K}$.

In comprehensive analysis of Figures 4(a) to 4(f), we find that no matter how to match subdomain and its boundary domain, the maximum deviation between the DRMC solutions and the final numerical solution appear near the real boundary, while the results in the central region are in good agreement; that is due to the boundaries of the present examples being gray, the artificial boundary surfaces of the subdomain are processed as blackbody surfaces in DRMC; the radiative energy transmitted to the artificial boundaries should be the energy passing to the discrete domains outside of the subdomains; if there are real boundary surfaces existing near the blackbody boundaries, this part of energy would be transmitted back to the subdomains by scattering, and when the optical thickness of the subdomain reduced, the radiative energy transmitted to the blackbody boundary surfaces would increase, which lead to deviation increase of the two methods near the boundary surfaces; however, if the boundary surfaces are transparent, the deviation would be eliminated, and the optical thickness can be further reduced.

3.1.2. Comparison and Analysis of Computing Time. Table 1 shows the comparison of computing time between TMC and DRMC in absorbing medium when predicting the radiative equilibrium temperature field. As is shown in Table 1, the computing time by DRMC is far less than that by TMC under the same sampling bundles. When optical thickness of the subdomain is greater than 2.0 , the calculating deviation of both methods is less than $1 \%$, while the maximum computing time decrease is $63.9 \%$ (optical thickness of subdomain is 2.0 ), and the minimal reduction is $32.6 \%$ (optical thickness of subdomain is 5.0 ) by DRMC.

3.1.3. Influences of the Sampling Number. Figure 5 shows the comparison of radiative equilibrium temperature fields calculated by DRMC and TMC with different sampling bundles and subdomain optical thickness $\tau_{z}$. When $\tau_{z}$ is greater than 2 , the grid numbers and the sampling mode are the same; if the number of sampling bundles reduced, the radiative equilibrium temperature field predicted by DRMC would be more close to the final numerical solution than that predicted by TMC; that is to say, the radiative equilibrium temperature field predicted by DRMC converges faster than that by TMC, because of DRMC limit sample tracking domain to the subdomain; to compare with TMC, the tracking domain reduced; thus less sampling bundles are required to get a reasonable statistical result in DRMC; if the sampling number reduced, the results predicted by DRMC will be closer to the final numerical solutions; in addition, because the DRMC is more efficient than TMC, the high computing efficiency helps convergence speed improvement. This trend is slowing down when optical thickness of the subdomain is less than 2.0. In summary, the results predicted by DRMC are still smoother and closer to the final solution than that by TMC.

3.2. Absorbing-Scattering Medium. For different subdomain and its boundary domain space, the radiative equilibrium temperature field of the absorbing-scattering medium with extinction coefficient $\kappa_{e}=15$ and scattering albedo $\omega=0.5$ are comparatively analyzed by DRMC.

3.2.1. Analysis of Subdomain and Boundary Domain. Figure 6 shows the temperature distribution of the absorbingscattering medium predicted by TMC method with different sampling bundles. It can be seen that the temperature field has converged when the number of sampling bundles is $N_{r}=10^{5}$; there is no obvious effects on the predicted temperature field by increasing the number of sampling bundles. In this paper, we consider that the temperature field 


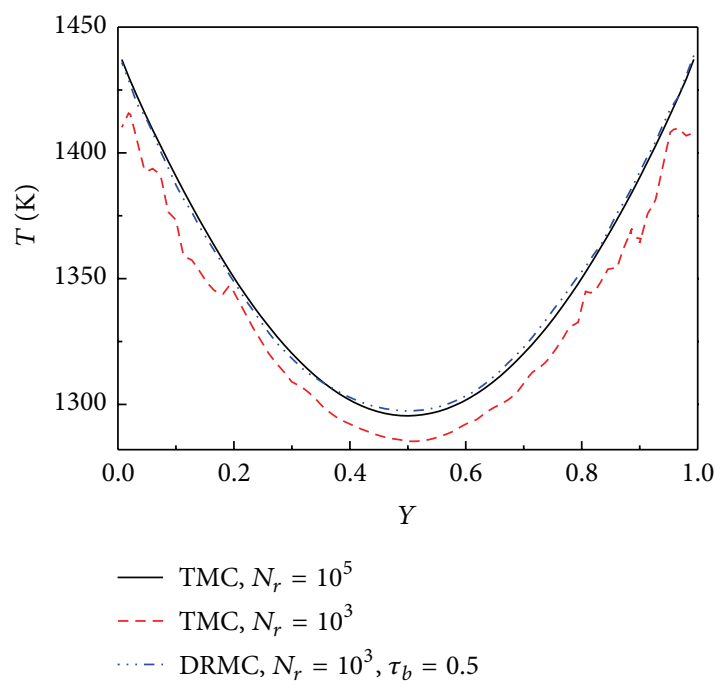

(a) $\tau_{z}=5.0, \tau_{b}=0.5$

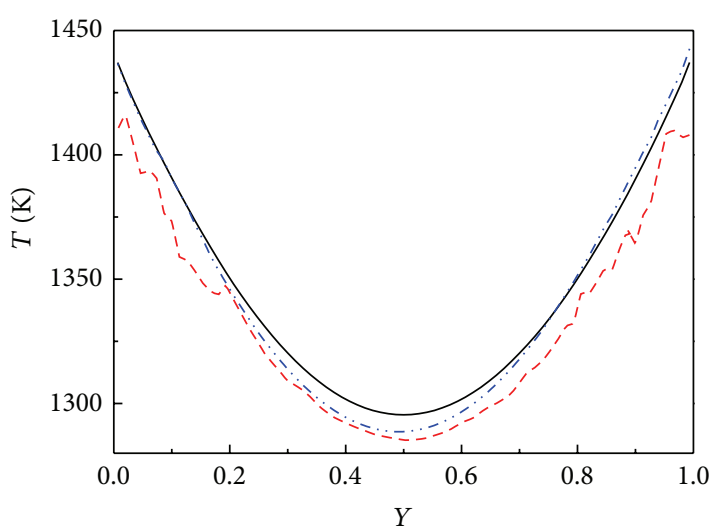

- TMC, $N_{r}=10^{5}$
-- TMC, $N_{r}=10^{3}$
$\cdots-$ DRMC, $N_{r}=10^{3}, \tau_{b}=0.5$

(c) $\tau_{z}=2.0, \tau_{b}=0.5$

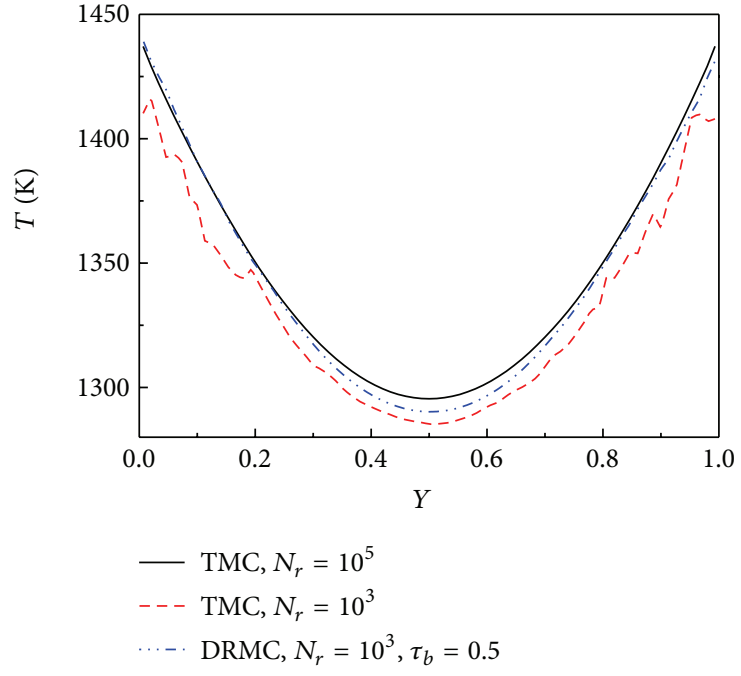

(b) $\tau_{z}=3.0, \tau_{b}=0.5$

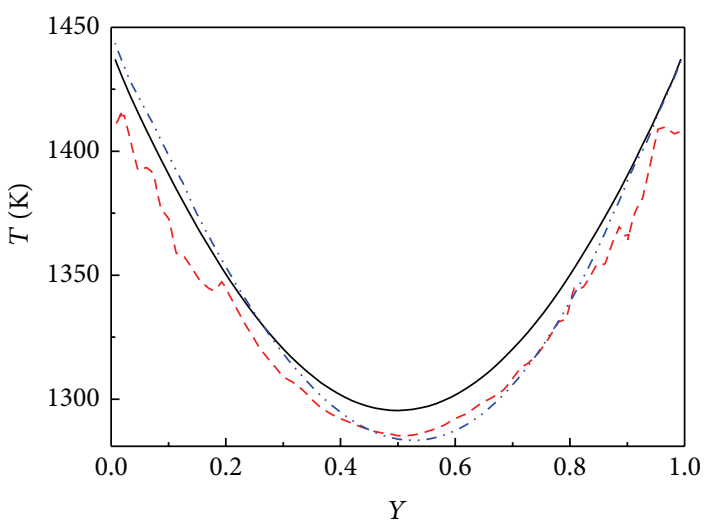

- TMC, $N_{r}=10^{5}$
--- TMC, $N_{r}=10^{3}$
$\cdots-$ DRMC, $N_{r}=10^{3}, \tau_{b}=0.5$

(d) $\tau_{z}=1.5, \tau_{b}=0.5$

FIgure 5: Comparison of temperature field of position $X=0.3$ predicted by DRMC MC and TMC in absorbing medium with different sampling number and $\tau_{z}$.

calculated by TMC is the final numerical solution when the sampling number of the unit is $N_{r}=10^{5}$. In the next section, we will compare the temperature results calculated by TMC and DRMC with different subdomain and boundary domain optical thicknesses, but with the same sampling numbers of $N_{r}=10^{5}$; see Figure 7.

Figure 7(a) shows that when subdomain optical thickness is $\tau_{z}=5$, compared with absorbing medium, the temperature field predicted by DRMC deviates from the final numerical solution under different $\tau_{b}$, the maximum deviation occurs in the center region of the medium, the value is $0.25 \%$, when $\tau_{b}=0.5$, and the maximum absolute temperature difference of the medium is about $3.3 \mathrm{~K}$. When $\tau_{z}$ reduced to 3 , the deviation increases with the reduction of $\tau_{b}$, but the maximum deviation does not exceed $0.34 \%$, and the absolute temperature deviation of the medium does not exceed $4.1 \mathrm{~K}$.
As $\tau_{z}$ decreased to 2.5 , when $\tau_{b}=2$, the temperature field predicted by DRMC and the final numerical solution would have the maximum deviation of $1.2 \%$; the maximum absolute temperature deviation reaches $14.7 \mathrm{~K}$, while when $\tau_{b}=0.5$, the maximum deviation is just $0.56 \%$, and the maximum absolute temperature deviation is $6.5 \mathrm{~K}$. Therefore, the scope of the boundary domain is not the bigger, the more reasonable. When $\tau_{z}=2$, according to setting $\tau_{b}$ reasonably, the deviation of temperature field predicted by DRMC and TMC may not exceed $0.44 \%$, and the maximum temperature difference does not exceed $6.2 \mathrm{~K}$.

Figure 7(e) has shown the results when $\tau_{z}=1.5$; it can be seen that the deviation of the temperature fields separately predicted by TMC and DRMC does not exceed $0.7 \%$ if the value of $\tau_{b}$ is reasonably set, and the deviation of absolute temperature predicted by the two methods does not exceed 
TABLE 2: Comparison of computational time of temperature fields predicted by TMC and DRMC in absorbing-scattering medium.

\begin{tabular}{|c|c|c|c|c|}
\hline Method & Sampling number & $\tau_{z}$ & $\tau_{b}$ & Computing time \\
\hline TMC & $N_{r}=10^{5}$ & & & $139 \mathrm{~min}$ and $11 \mathrm{~s}$ \\
\hline \multirow{6}{*}{ DRMC } & \multirow{6}{*}{$N_{r}=10^{5}$} & 5.0 & 0.2 & $105 \mathrm{~min}$ and $55 \mathrm{~s}$ \\
\hline & & 3.0 & 0.2 & $95 \mathrm{~min}$ and $35 \mathrm{~s}$ \\
\hline & & 2.5 & 0.2 & $87 \mathrm{~min}$ and $43 \mathrm{~s}$ \\
\hline & & 2.0 & 0.2 & $82 \mathrm{~min}$ and $17 \mathrm{~s}$ \\
\hline & & 1.5 & 0.2 & $76 \mathrm{~min}$ and $04 \mathrm{~s}$ \\
\hline & & 1.0 & 0.2 & $71 \mathrm{~min}$ and $43 \mathrm{~s}$ \\
\hline
\end{tabular}

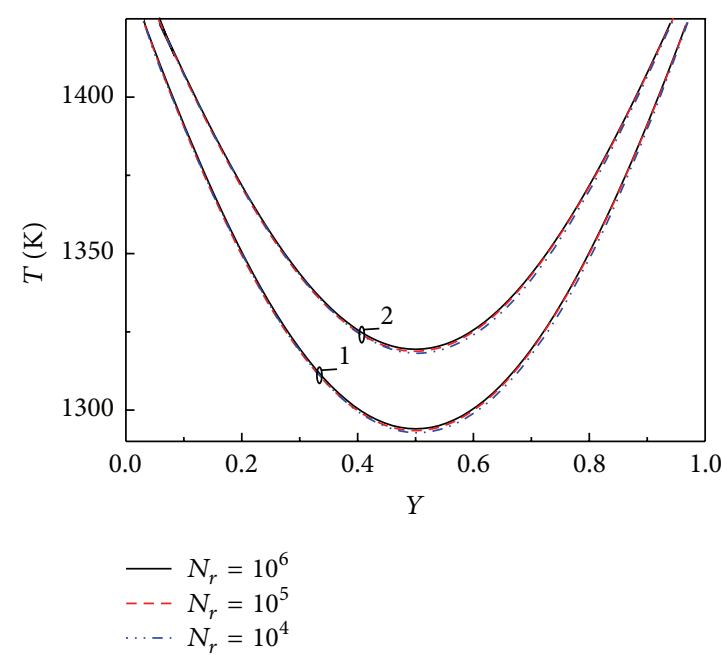

(a) Curve $1: X=0.3 ; 2: X=0.5$

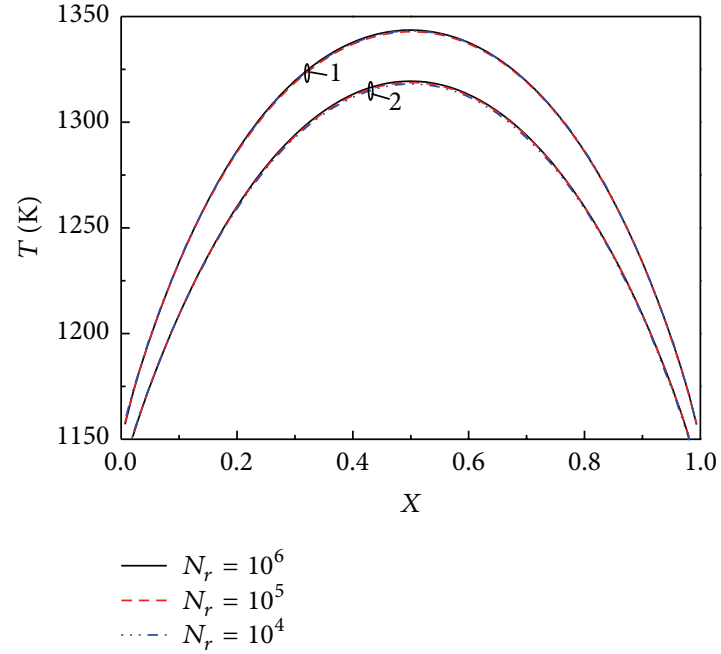

(b) Curve $1: Y=0.3 ; 2: Y=0.5$

FIGURE 6: Temperature field in absorbing-scattering medium predicted by TCM method.

9.8 K. Figure 7(f) shows the comparison of predicted temperature field by DRMC and the final numerical solution with different $\tau_{b}$ and $\tau_{z}=1$. For different $\tau_{b}$, the deviation between the temperature field predicted by DRMC and the final numerical solution is obvious, and no matter how $\tau_{b}$ changed, the deviation between the temperature field predicted by TMC and DRMC is over $1 \%$; when $\tau_{b}=0.5$, the deviation of temperature field predicted by the two method still reaches $1.1 \%$; the absolute temperature difference is about $17.3 \mathrm{~K}$.

In comprehensive analysis of Figures 7(a) to 7(f), it can be seen that if the boundary domain optical thickness is reasonably selected, when $\tau_{z}$ is less than 5 , there is just little difference between the temperature field predicted by DRMC and TMC in absorbing-scattering medium. If $\tau_{z}$ is greater than 2 , the deviation of the radiative equilibrium temperature predicted by TMC and DRMC does not exceed $0.7 \%$; in general, the deviation would increase with the decrease of $\tau_{z}$ in absorbing-scattering medium.

3.2.2. Comparison of Computing Time. Table 2 shows the comparison of computing time between TMC and DRMC in absorbing and scattering medium when predicting the radiative equilibrium temperature field. The computing time by DRMC is far less than that by TMC under the same sampling numbers. As have been analyzed, the calculation deviation of the two methods is less than $1.0 \%$ when the optical thickness of subdomain is greater than 2.0, while the computing time of the two methods is reduced by $40.9 \%$ at the same time (optical thickness of subdomain is 2.0), and the minimal reduction of computing time is $24.5 \%$ (optical thickness of subdomain is 5.0).

3.2.3. Influences of Sampling Number. Figure 8 shows the comparison of radiative equilibrium temperature field in the absorbing-scattering medium predicted by DRMC and TMC with different sampling numbers and subdomain optical thickness $\tau_{z}$. The figures show that when $\tau_{z}$ is greater than 2 , the computing grids and the sampling mode are the same; the radiative equilibrium temperature field predicted by DRMC would be closer to the final numerical solution than that predicted by TMC if the sampling bundles reduced. When $\tau_{z}$ is less than 2.0, with the decrease of sampling number, both methods are deviated from the final numerical solution; the advantage of DRMC is not so obvious.

\section{Conclusions}

By the proposed DRMC method, the radiative transfer in a two-dimensional rectangular medium is simulated in this paper; the medium can be absorbing or absorbing-scattering. 


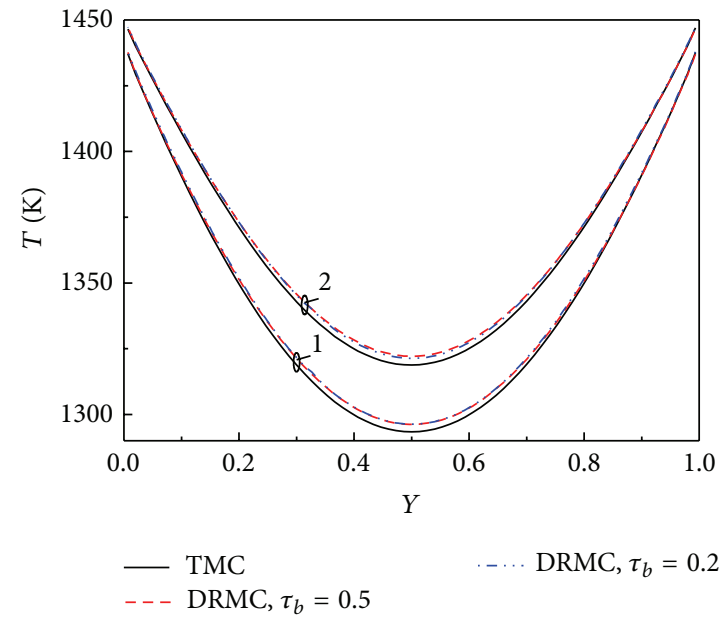

(a) $\tau_{z}=5.0$
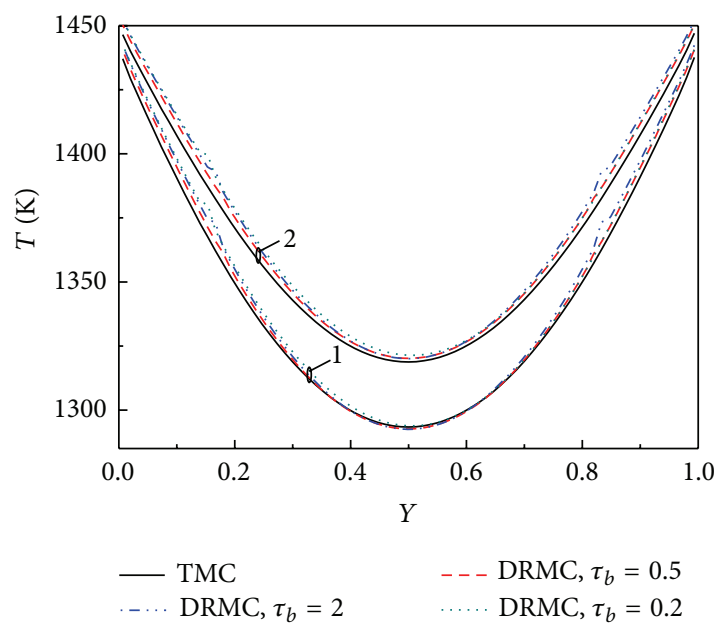

(c) $\tau_{z}=2.5$

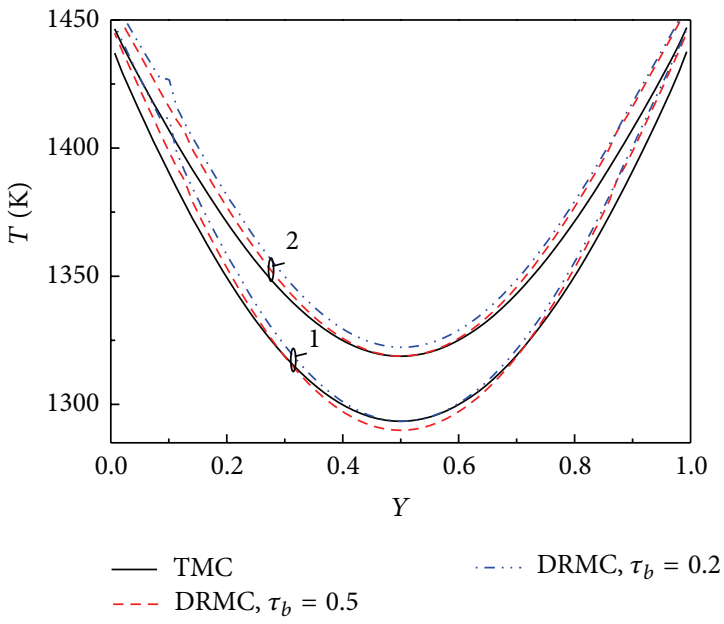

(e) $\tau_{z}=1.5$

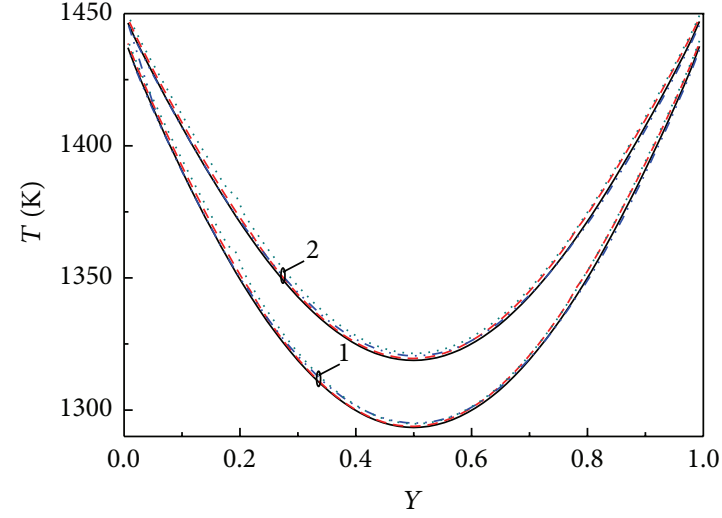

— TMC

-- DRMC, $\tau_{b}=0.5$

DRMC, $\tau_{b}=0.2$

(b) $\tau_{z}=3.0$

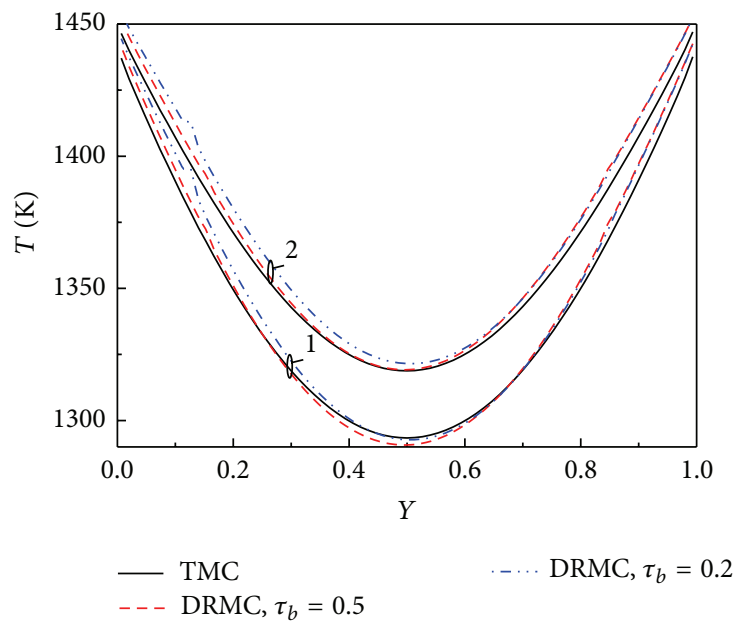

(d) $\tau_{z}=2.0$

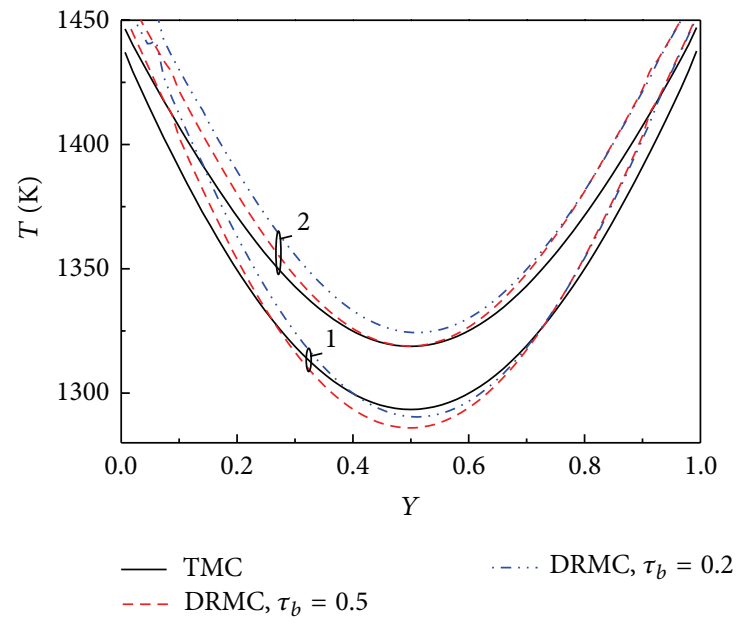

(f) $\tau_{z}=1.0$

FIGURE 7: Comparison of temperature fields in absorbing-scattering medium predicted by TMC and DRMC, Curve $1: X=0.3 ; 2: X=0.5$. 

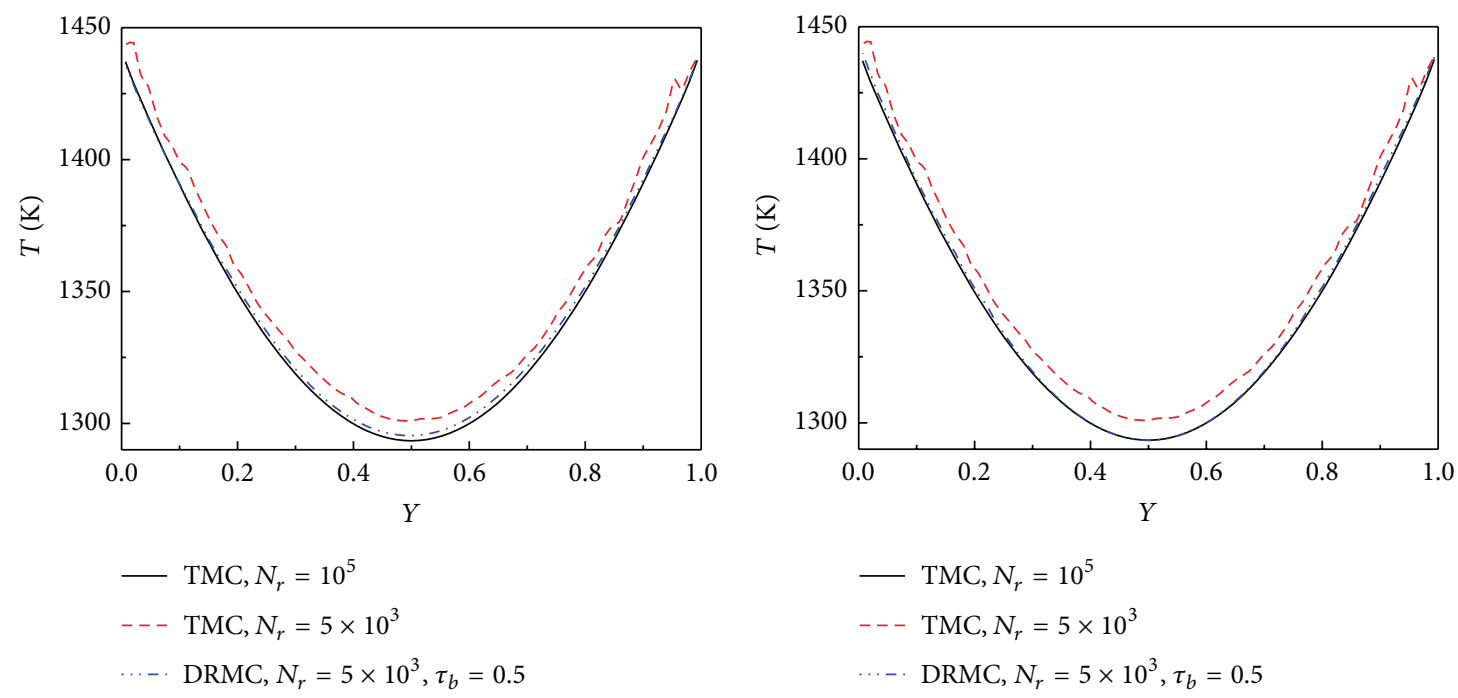

(a) $\tau_{z}=5.0$ and $\tau_{b}=0.5$

(b) $\tau_{z}=3.0$ and $\tau_{b}=0.5$
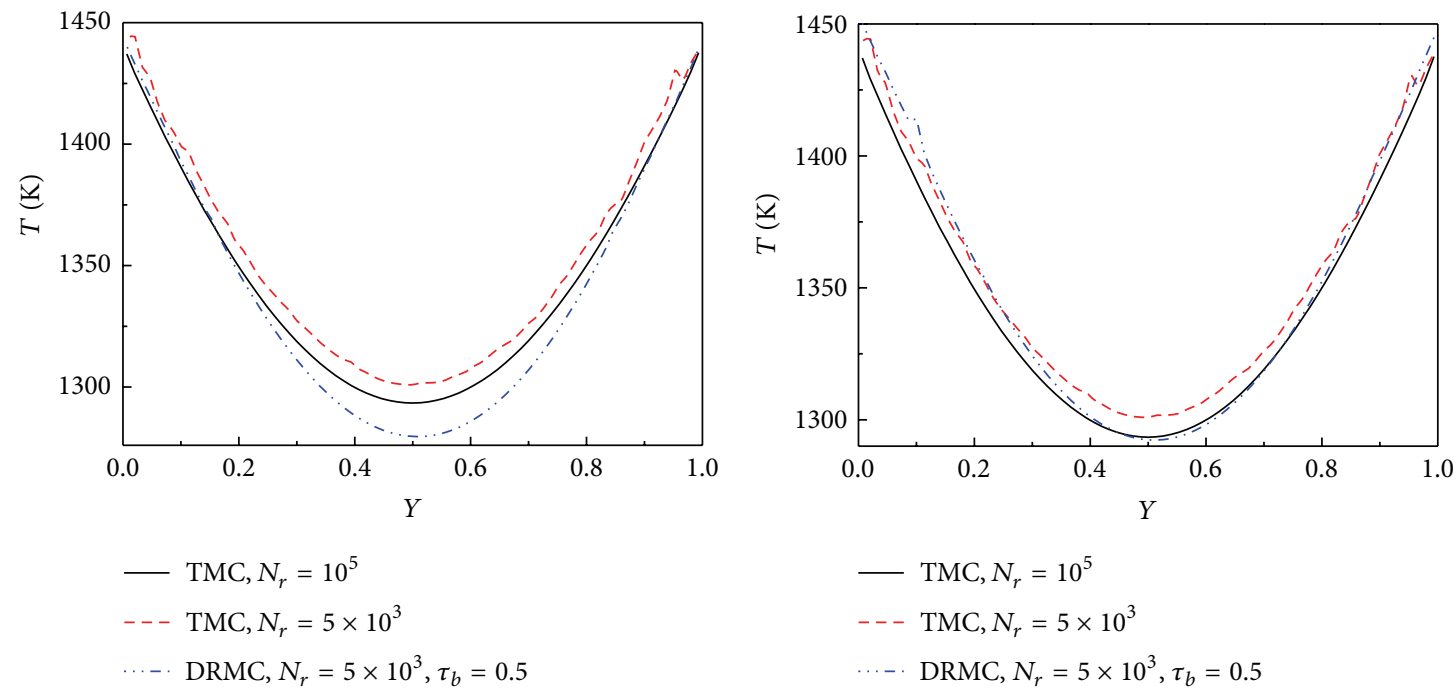

(c) $\tau_{z}=2.0$ and $\tau_{b}=0.5$

(d) $\tau_{z}=1.5$ and $\tau_{b}=0.5$

FIgURE 8: Comparison of temperature field of position $X=0.3$ predicted by DRMC MC and TMC in absorbing medium with different sampling number and $\tau_{z}$.

The predicted radiative equilibrium temperature fields by DRMC are in good agreement with the final numerical solution; this indicates that our DRMC model is creditable.

For radiative transfer in optically thick participating medium, most of the bundles will be absorbed by the medium in the vicinity of their emission positions; it means that only a few bundles participate in distant radiative transfer; therefore, a large number of bundles are required in order to get a creditable statistical solution. In addition, to obtain high precision solution, more grid numbers are needed in the medium with large optical thickness. Therefore, to simulate radiative transfer in such optically thick medium by TMC method, the large storage capacity and the long computing time must be accepted. The method of DRMC by region adaption limit sample tracking domain within a certain optical thickness centered about the bundle's current position, while the radiative energy transmit to the discrete elements outside of the subdomain is equivalently calculated by setting an equivalent temperature region reasonably, in DRMC, it just needs to store radiation exchange factor of the subdomain, and therefore, to compare with TMC, DRMC can reduce storage capacity and computing time.

The present DRMC method can expand the calculation range of the TMC method when simulating radiation heat transfer in participating medium with large optical thickness; if the medium is optically thin, the optical thickness of the 
medium is less than subdomain optical thickness, and the DRMC would devolve back to TMC.

In this paper, we simulated radiative transfer in a twodimensional rectangular participating medium with optical thickness of $\tau=\kappa_{e} L_{x}=\kappa_{e} L_{y}=15$ and scattering albedo $\omega=0.5$ (if the medium is absorbing-scattering) by DRMC; the results are compared with those predicted by TMC method; the following conclusions can be drawn.

(1) In absorbing medium, if the optical thickness of calculation subdomain is greater than 2 , the deviation of temperature field predicted by DRMC and the final numerical solution is less than $1 \%$ under the same sampling bundles.

(2) According to comparison and analysis of the temperature fields in absorbing and/or scattering medium predicted by DRMC and TMC method, if the sampling bundles reduced, the results predicted by DRMC can be closer to the final numerical solution than that predicted by TMC under the same sampling bundles; this means that under the same calculation accuracy requirements DRMC can achieve the purpose of improving the solving efficiency by reducing the sampling bundles.

(3) When solving radiative equilibrium problems in participating medium with the same computational parameters, DRMC is much efficient than TMC. In our calculation examples in this paper, DRMC can save $24.5 \%$ to $63.9 \%$ computing time when the subdomain optical thickness is 2 to 5 . In addition, one thing should be mentioned is that the larger the optical thickness of the medium is, the better the DRMC will display its advantages of saving storage capacity and computing time.

\section{Conflict of Interests}

The authors declare that there is no conflict of interests regarding the publication of this paper.

\section{Acknowledgment}

The authors are indebted to the financial supports by the National Natural Science Foundation of China (Grant no. 51106036, no. 51176038, and no. 51276050).

\section{References}

[1] R. Viskanta, "Overview of convection and radiation in high temperature gas flows," International Journal of Engineering Science, vol. 36, no. 12-14, pp. 1677-1699, 1998.

[2] J. R. Howell, "The Monte Carlo method in radiative heat transfer," Journal of Heat Transfer, vol. 120, no. 3, pp. 547-560, 1998.

[3] P.-F. Hsu and J. T. Farmer, "Benchmark solutions of radiative heat transfer within nonhomogeneous participating media using the Monte Carlo and YIX method," Journal of Heat Transfer, vol. 119, no. 1, pp. 185-188, 1997.
[4] L. M. Ruan and H. P. Tan, "Solutions of radiative heat transfer in three-dimensional inhomogeneous, scattering media," Journal of Heat Transfer, vol. 124, no. 5, pp. 985-988, 2002.

[5] V. Eymet, R. Fournier, S. Blanco, and J. L. Dufresne, "A boundary-based net-exchange Monte Carlo method for absorbing and scattering thick media," Journal of Quantitative Spectroscopy and Radiative Transfer, vol. 91, no. 1, pp. 27-46, 2005.

[6] S. T. Surzhikov and J. R. Howell, "Monte Carlo simulation of radiation in scattering volumes with line structure," Journal of Thermophysics and Heat Transfer, vol. 12, no. 2, pp. 278-281, 1998.

[7] A. de Lataillade, J. L. Dufresne, M. El Hafi, V. Eymet, and R. Fournier, "A net-exchange Monte Carlo approach to radiation in optically thick systems," Journal of Quantitative Spectroscopy and Radiative Transfer, vol. 74, no. 5, pp. 563-584, 2002.

[8] H. T. Yu, D. Liu, Y. Y. Duan, and X. D. Wang, "Theoretical model of radiative transfer in opacified aerogel based on realistic microstructures," International Journal of Heat and Mass Transfer, vol. 70, pp. 478-485, 2014.

[9] J. Fricke, U. Heinemann, and H. P. Ebert, "Vacuum insulation panels-from research to market," Vacuum, vol. 82, no. 7, pp. 680-690, 2008.

[10] H. Wu, Y. Liao, Y. Ding, H. Wang, C. Peng, and S. Yin, "Engineering thermal and mechanical properties of multilayer aligned fiber-reinforced aerogel composites," Heat Transfer Engineering, vol. 35, no. 11-12, pp. 1061-1070, 2014.

[11] F. Enguehard, "Multi-scale modeling of radiation heat transfer through nanoporous superinsulating materials," International Journal of Thermophysics, vol. 28, no. 5, pp. 1693-1717, 2007.

[12] M. Duan and Q. Min, "A semi-analytic technique to speed up successive order of scattering model for optically thick media," Journal of Quantitative Spectroscopy and Radiative Transfer, vol. 95, no. 1, pp. 21-32, 2005.

[13] A. Amosov, G. Panasenko, and B. Rutily, "An approximate solution to the integral radiative transfer equation in an optically thick slab," Comptes Rendus Mecanique, vol. 331, no. 12, pp. 823828, 2003.

[14] J. A. Fleck Jr. and E. H. Canfield, "A random walk procedure for improving the computational efficiency of the implicit Monte Carlo method for nonlinear radiation transport," Journal of Computational Physics, vol. 54, no. 3, pp. 508-523, 1984.

[15] J. Giorla and R. Sentis, "A random walk method for solving radiative transfer equations," Journal of Computational Physics, vol. 70, no. 1, pp. 145-165, 1987.

[16] N. A. Gentile, "Implicit Monte Carlo diffusion-an acceleration method for Monte Carlo time-dependent radiative transfer simulations," Journal of Computational Physics, vol. 172, no. 2, pp. 543-571, 2001.

[17] J. D. Densmore, T. J. Urbatsch, T. . Evans, and M. W. Buksas, "A hybrid transport-diffusion method for Monte Carlo radiativetransfer simulations," Journal of Computational Physics, vol. 222, no. 2, pp. 485-503, 2007.

[18] J. D. Densmore, K. G. Thompson, and T. J. Urbatsch, "A hybrid transport-diffusion Monte Carlo method for frequencydependent radiative-transfer simulations," Journal of Computational Physics, vol. 231, no. 20, pp. 6924-6934, 2012.

[19] M. Cherkaoui, J. L. Dufresne, R. Fournier, J. Y. Grandpeix, and A. Lahellec, "Monte Carlo simulation of radiation in gases with a narrow-band model and a net-exchange formulation," Journal of Heat Transfer, vol. 118, no. 2, pp. 401-407, 1996. 
[20] L. M. Ruan, H. Qi, L. H. Liu, and H. P. Tan, “The radiative transfer in cylindrical medium and partition allocation method by overlap regions," Journal of Quantitative Spectroscopy and Radiative Transfer, vol. 86, no. 4, pp. 343-352, 2004.

[21] L. M. Ruan, L. H. Liu, and H. P. Tan, "The study on approximating the open boundary of two-dimension medium as one black wall," Heat and Mass Transfer, vol. 40, no. 3-4, pp. 319-324, 2004. 


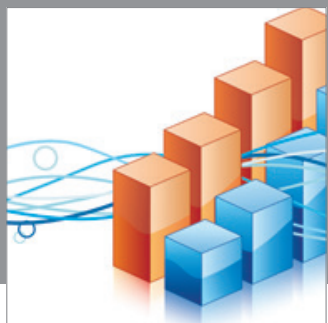

Advances in

Operations Research

mansans

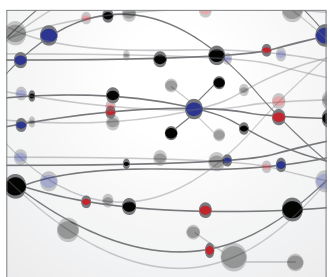

The Scientific World Journal
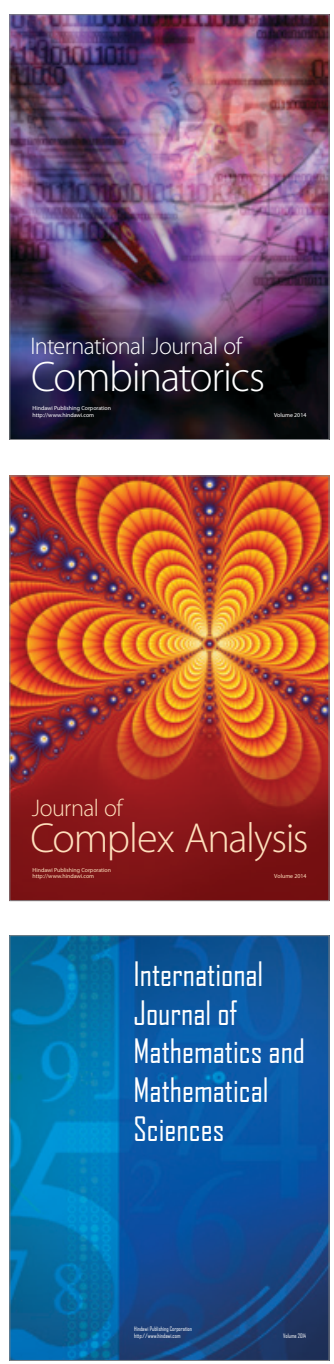
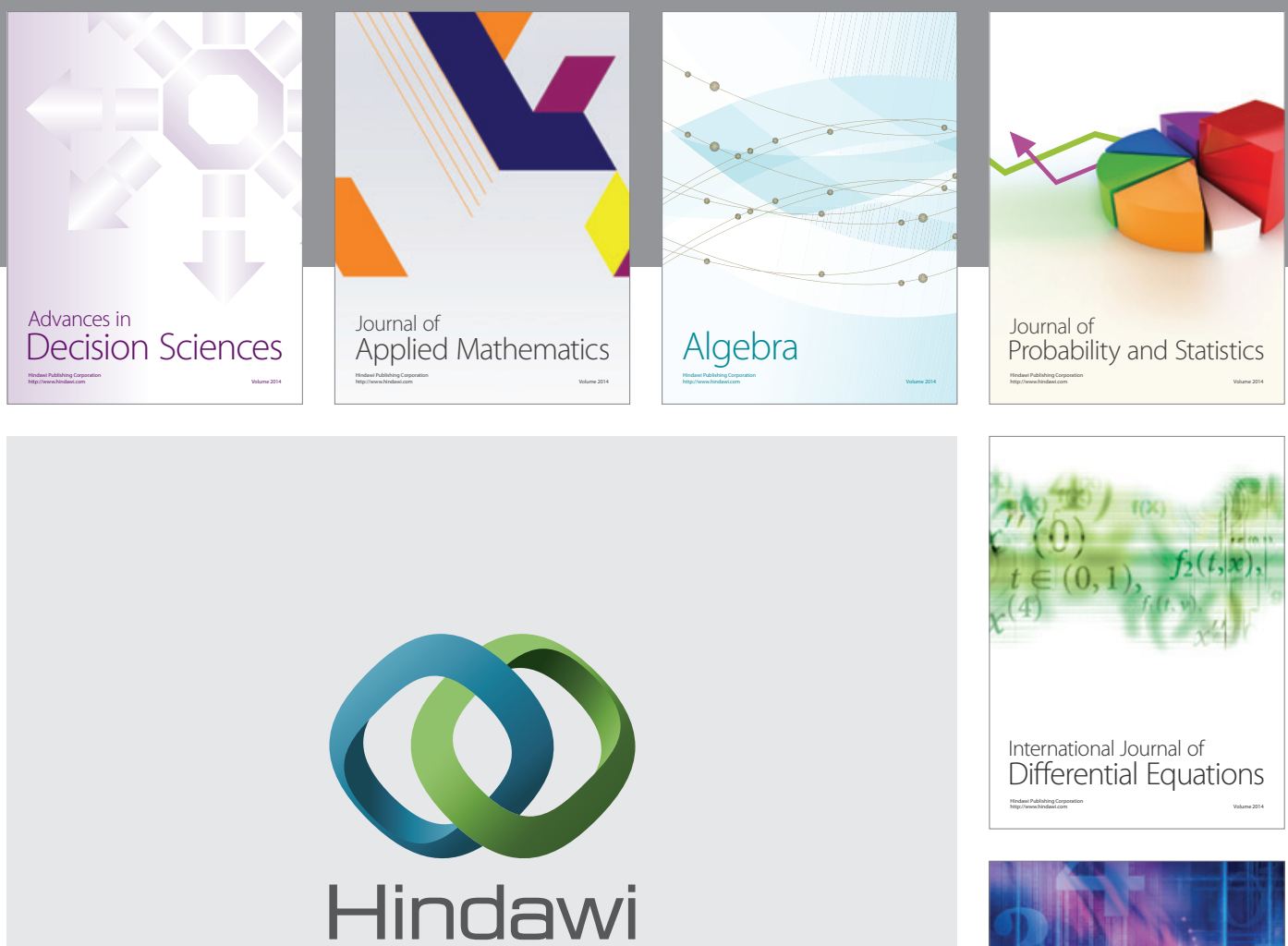

Submit your manuscripts at http://www.hindawi.com
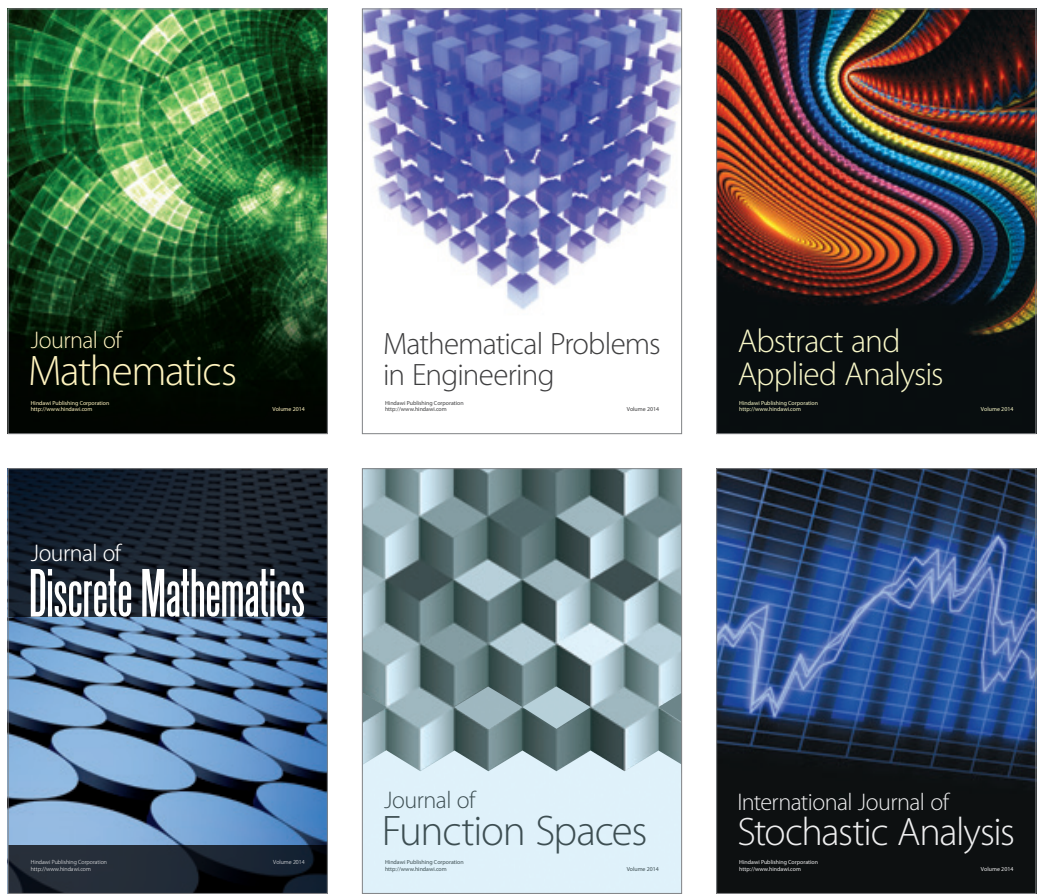

Journal of

Function Spaces

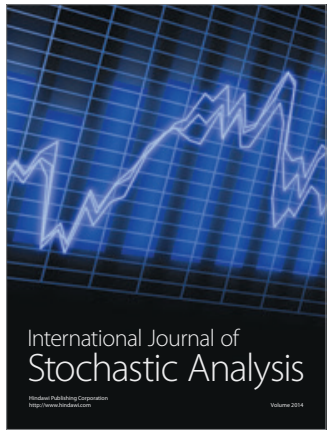

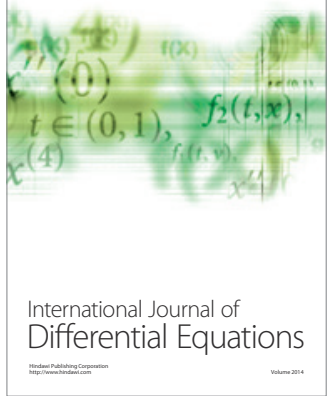
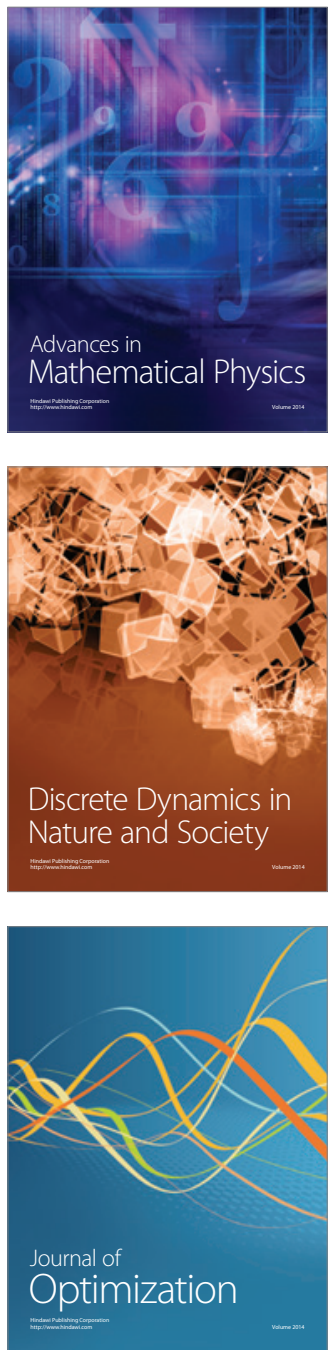